Editorial

\title{
Plagiarism in Healthcare Education
}

\author{
Jugal Kishore', Charu Kohli ${ }^{2}$
}

${ }^{1}$ Director Professor and Head, Community Medicine, VMMC and Safdarjung Hospital, New Delhi.

${ }^{2}$ Senior Technical Officer, IPE Global Limited.

DOI: https://doi.org/10.24321/2455.9199.201901

\section{I $\quad \mathbf{N} \quad \mathbf{F} \quad \mathbf{O}$}

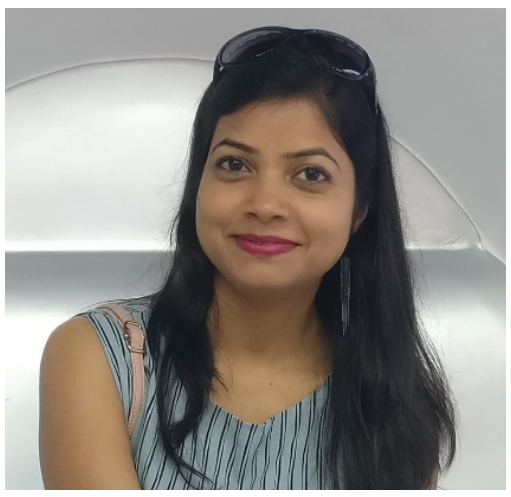

Corresponding Author:

Charu Kohli, IPE Global Limited.

E-mail Id:

kohlicdoc17@gmail.com

Orcid Id:

https://orcid.org/0000-0001-9069-5173

How to cite this article:

Kishore J, Kohli C. Plagiarism in Healthcare Education. Int J HealthCare Edu \& Med Inform 2019; 6(1): 1-2.

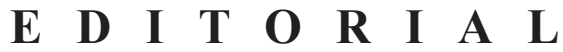

The misappropriation of other's intellectual contribution is plagiarism. It is considered to be a serious form of research misconduct. It can be said that it is one of the most frequently reported type of research misconduct. ${ }^{1}$ According to the Oxford English Dictionary, the plagiarism is defined as 'the practice of taking someone else's work or ideas and passing them off as one's own'. Perrin, Larkham and Culwin define plagiarism as the use of an author's words, ideas, reflections and thoughts without proper acknowledgment of the author. ${ }^{2-4}$

Although plagiarism has been reported at all stages of career of a researcher but it is most frequently reported in early career scientists. ${ }^{5}$ Academic misconduct among medical students has increased with advent of technology owing to easy availability of literature on internet which could be copied and modified. ${ }^{6}$

There are several factors responsible for plagiarism in research like insufficient sensitization in research integrity and code of ethics, lack of focus on developing research especially writing skills, high degree of tolerance or lack of stringent rules and regulations in misconduct, no institutional policies specific to detect and prevent plagiarism, lack of awareness towards use of plagiarism detecting software, rampant corruption in professional growth and opportunities and inappropriate criteria of quantity of publications in career growth and promotions. ${ }^{7-10}$ Therefore, removal of plagiarism is difficult task till comprehensive policy involving all sectors of healthcare and research are not frame.

A cross-sectional study was carried out among 400 undergraduate medical and nursing students in Iraq. Data on plagiarism was collected using a self-administered questionnaire. The prevalence of plagiarism was very high upto 54.3\%. Plagiarism was perceived more among medical students as compared to nursing students (58.9\% versus $43.3 \%$; $(P=0.004)$. More than one third of the students did not know what plagiarism was, and only $28 \%$ were aware of the legal consequences of plagiarism. Reported causative factors for plagiarism were the ease with which others' work could be plagiarized, confusion, cultural reasons and pressure to meet deadlines. ${ }^{11}$

Another study was conducted to assess the knowledge and attitude regarding plagiarism among post graduate dental students of Bangalore city, India. Results stated that $41.2 \%$ of them were having very good 
knowledge followed by $40.5 \%$ with good knowledge and only $18.4 \%$ with excellent knowledge about plagiarism. $87.8 \%$ had uncertain attitude, followed by $9.7 \%$ with positive attitude and only $2.4 \%$ with negative attitude towards prevention of plagiarism. Strong positive correlation was seen between overall knowledge and age $(r=0.90$, $p=0.01$ ), week positive correlation was seen between overall knowledge and academic year $(r=0.56, p=0.01) .{ }^{12}$

Training of medical undergraduate students is key to inculcate research ethics among future generations. It is essential to maintain research integrity and prevent plagiarism. There should be dedicated seminars on plagiarism during undergraduate and postgraduate studies in all teaching institutions. Workshops of healthcare research ethics should be designed in such a manner which can bring changes in attitude. An oath regarding prevention of plagiarism at the time of graduation could be considered. Institutions could run short distance learning courses or e-learning modules to prevent and detect plagiarism.

Detection of plagiarism is crucial to maintain quality of healthcare research. There is a need to find prevention strategies and to adopt a comprehensive approach to raise awareness about misappropriation in use of intellectual property.

More studies are required in Indian settings to determine the specific reasons behind plagiarism and methods to foster awareness among medical students about plagiarism. It would help prevent future cases of research misconduct. At the same time, senior faculty should provide support and guidance to students' on research integrity.

\section{References}

1. Smith R. What is research misconduct. Journal of the Royal College of Physicians of Edinburgh 2000; 30: 4-8.

2. Perrin R. Pocket guide to APA style. $3^{\text {rd }}$ ed. Boston, MA: Wadsworth; 2009.

3. Larkham M. Plagiarism and its treatment in higher education. Journal of Further and Higher Education 2002; 26(4): 339-349.

4. Culwin F. Plagiarism issues for higher education. Vine 2001; 31(2): 36-41.

5. Martinson BC, Anderson MS, de Vries R. Scientists behaving badly. Nature 2005; 435(7043): 737-738.

6. Mortaz Hejri S, Zendehdel K, Asghari F et al. Academic disintegrity among medical students: a randomised response technique study. Med Educ 2013; 47(2): 144-153.

7. Davis MS. The role of culture in research misconduct. Accountability in Research 2003; 10(3): 189-201.

8. Cameron $\mathrm{C}$, Zhao H, McHugh MK. Publication ethics and the emerging scientific workforce: Understanding
"Plagiarism" in a global context. Academic Medicine 2012; 87(1): 51-54.

9. Heitman $\mathrm{E}$, Olsen $\mathrm{CH}$, Anestid ou L et al. New graduate students' baseline knowledge of the responsible conduct of research. Academic Medicine 2007; 82(9): 838-845.

10. Vasconcelos S, Leta J, Costa L et al. Discussing plagiarism in Latin American science. EMBO Reports 2009; 10(7): 677-682.

11. Ismail KH. Perceptions of Plagiarism Among Medical and Nursing Students in Erbil, Iraq. Sultan Qaboos Univ Med J 2018; 18(2): e196-e201.

12. Naveen N, Raveendran N, Vanishree $\mathrm{N}$ et al. An effectual analytics and cross sectional study on plagiarism among dental post graduates of Bangalore city. Int J Applied Dental Sci 2017; 3(3): 23-26. 